form filaments in vitro. The assembly products were analyzed by electron microscopy. We observed that full-length filensin and the $50 \mathrm{kDa}$ form of filensin coassemble with phakinin into long filaments with a width of $15-20 \mathrm{~nm}$, even though globular particles were not evident. In contrast, the $38 \mathrm{kDa}$ protein did not form filaments but gave only aggregates. These results suggest that the tail domain of filensin is critically involved in filament assembly and that acceleration of processing during cataractogenesis leads to a loss of function of BFs in lens fiber cells.

\section{P-051 天虫由来ヤママリンの構造・活性相関}

Structural characterization of a diapause-related peptide from the wild silkmoth Keisuke Oyauchi (1), Masakatsu Kamiya (1), Takuya Yokoyama (2), Wang Mofei (2), Tomoyasu Aizawa (2), Makoto Demura (1), Koichi Suzuki (3), Keiichi Kawano (2). (1) Graduate School of Life Sciences, Hokkaido University; 2: Graduate School of Sciences, Hokkaido University; 3: Faculty of Agriculture, Iwate University)

Yamamarin, a pentapeptide with amidated C-terminus (DILRG-NH$)$ ), has been isolated from diapausing pharate first instar larvae of the wild silkmoth Antherae yamamai. Yamamarin is believed to be responsible for the regulation of diapause but the pattern of its production, its source, and its exact role in dispausing insect remain to be analyzed. Recently, Dr. Suzuki and his co-workers found that this peptide significantly suppresses proliferation of rat hepatoma (liver cancer) cells. Moreover, they found that a palmitonyl-conjugated derivative (C16-DILRG-NH causes reversible growth arrest in the rat hepatoma cells and inhibits embryonic development in the silkworm, Bombyx mori. The results indicate that Yamamarin and the derivatives are a promising candidate for therapeutic use and vermicide. However, its action mechanisms remain unclear.

As a first step toward elucidating the mechanism of growth suppression of rat hepatoma cells, we investigated the structural characteristic of Yamamrin and the mutants by CD and NMR spectroscopy. Cell proliferation assay of a series of alanine mutants indicated that amino acid residues 3-5 of Yamamarin is important for growth suppression. CD and NMR results indicate that these peptides adopt random coil structure in water. In this presentation, we will describe the structura properties of Yamamarin and discuss its relationship to activity.

\section{P-052 分子動力学シミュレーションによるPhoB DNA 結合タンパク質側鎖 のダイナミクス解析}

Analysis of side-chain dynamics of PhoB DNA binding/transactivation domain using molecular dynamics simulations

Tsutomu Yamane (1), Hideyasu Okamura (2), Yoshifumi Nishimura (1), Akinori Kidera (1,3) Mitsunori Ikeguchi (I) (1:International Graduate School of Arts and Sciences, Yokohama Cit University; 2: Biochemistry Department, National institute of agrobiological sciences; 3 . Research Program for Computational Science, RIKEN)

The PhoB DNA-binding/transactivation domain is a C-terminal functional domain of the PhoB for binding of the pho box, which is situated upstream of the pho regulon. The NMR structure of the DNA binding form of the PhoB DNA-binding/transactivation domain was solved by our group (PDB: 2z33) (1) In addition, backbone and side-chain dynamics of PhoB-DNA binding/transactivation domain were analyzed using the NMR relaxation sutud (2).In the present study, molecular dynamics (MD) simulations of the free-form and DNA-binding form of PhoB-DNA binding/transactivation domain were carried out, and resulting backbone and side-chain dynamics were compared with those of NMR relaxation experiments. The model-free order parameters for the backbone $\mathrm{N}-\mathrm{H}$ bond $\left(S_{N H}^{2}\right)$ and the methyl-averaging axis $\left(S_{\text {axis }}^{2}\right)$ obtained from the MD simulations were in agreement with experimental values. It was found that $S^{2}{ }_{N H}$ and $S_{a x i s}^{2}$ correlate well with the root-mean-square fluctuation (RMSF) of the backbone nitrogen atoms and the methyl carbon atoms obtained from the MD simulations, respectively, in contrast to weak correlations between the order parameters and crystal temperature factors. The $S_{a x i s}^{2}-$ RMSF plot showed the clear dependence of $S^{2}$ xris on amino-acid species and the positions of the methy groups in the side-chains, suggesting that dynamics of the methyl group in side chains are strongly affected by geometry of side chains in amino-acid species.(1) T. Yamane et al., Proteins 2008:71, 1970-1983.(2) H. Okamura et al., J. Mol. Biol. 2007:367, 1093-1117.

\section{P-053 Structural and Functional Studies on the conserved hypothetical protein HP0894 from Helicobacter pylori}

Structural and Functional Studies on the conserved hypothetical protein HP0894 from Helicobacter pylori

Ki-young Lee (1), Kyung-Doo Han (1), Atushi Matsuura (1), Ae-Ran Kwon (1), Sung-Jean Park (1), and Bong-Jin Lee (1)(1:Research Institute of Pharmaceutical Sciences, College of Pharmacy, Seoul National University)

As part of our structural genomics effort on Helicobacter pylori, we have determined solution structure of the 88-residue conserved hypothetical protein HP0894 from Helicobacter pylori using multidimentional heteronuclear NMR spectroscopy. In addition, in order to identify the biological role of HP0894, we investigated the interactions of HP0894 with HP0895 as a potential antitoxin and their functions. As results presented here, HP0894 binds to HP0895 strongly with the stoichiometry of 1:1. HP0894/HP0895 complex and HP0895 are large multimers, while HP0894 is a monomer. HP0894 shows in vitro RNase activity on mRNA, while HP0895 inhibits its activity. HP0894 expression has a toxic effect on Escherichia coli cell growth, but the co-expression of HP0895 neutralizes the toxicity of HP0894. These results, including sequence and structure analyses, suggest that HP0894 is a RelE/YoeB family toxin with intrinsic RNase activity and HP0895 works as its antitoxin.

\section{P-054 レーザーフラッシュフォトリシス法による節足動物由来へモシア ニンのアロステリック効果の研究}

Allosteric effect of arthropod hemocyanin studied by laser flash photolysis Naoki Tanaka(1),Satoshi Nagao(1),Takuya Yamada(1),Luigi Bubacco(2),Mariano Beltramini(2),Paolo Di Muro(2) and Shun Hirota(1).(1:Graduate School of Materials Science, Nara Institute of Science and Technology;2: Dept Biology, Univ of Padova)

Hemocyanin $(\mathrm{Hc})$ is an oxygen carrier and storage protein found in molluscs and arthropods. Significant differences are observed between mollusc and arthropod Hes in size of the functional units and in their tertiary and quaternary structures. Specifically, arthropod Hes are structurally homogenous oligomeric proteins with a minimal functional subunit of $75 \mathrm{kDa}$. Oxygen binding to arthropod Hes is very sensitive to $\mathrm{pH}$ and the relevance of this Bohr effect resides in the capability of these proteins to respond to a $\mathrm{pH}$ variation in the medium with a variation in affinity for molecular oxygen to meet physiological needs. Although $\mathrm{Hc}$ has been studied extensively, detailed information concerning oxygen binding kinetics and the origins of both cooperativity and the Bohr effect is still limited. OxyHc is blue and its optical spectrum is characterized by a CT band at about $345 \mathrm{~nm}$ and a d-d band at about $600 \mathrm{~nm}$. DeoxyHc is colorless. Taking advantage of these optical differences, flash photolysis was used to investigate the functional and structural effects of $\mathrm{pH}$ and lactate on the oxygen affinity of arthropod Hc.

\section{P-055 表面力測定による転写タンパク質の熱ストレス応答性の研究}

Study of thermally stressed response of transcription proteins by surface forces measurement.

Yoshiki Nakata (1), Takahiro Yamaguchi (1), Takehiro Suzuki (1), Kazuya Morikawa (2), Kazue Kurihara (1). (1: Tohoku Univ. IMRAM; 2: Tsukuba Univ. Basic Med.)

The interactions between proteins are essential for living systems. Recent study shows that the thermal stress modulates the transcription process of Staphylococcus aureus, however, its mechanism remains unclear. In this work, we investigated the effect of the thermal stress on the interactions between SigB, transcription factor of Staphylococcus aureus and RsbW, anti-SigB factor. They were immobilized on solid substrates with affinity tags, Glutathion-S-Transferase (GST) for SigB and Maltose Binding Protein (MBP) for RsbW. Their interactions were studied using colloidal probe atomic force microscopy, and structure were evaluated by IR-RAS. The heat treatment was conducted at $48{ }^{\circ} \mathrm{C}$ for $10 \mathrm{~min}$. Interactions The heat treatment for 0.5 and 1.0 min decreased the adhesion force between SigB and RsbW. For more than $1.0 \mathrm{~min}$ of the heat treatment, the adhesion force was increased to the value before heat treatment. It suggests that the influence of the heat treatment occurs in a short time. Structure evaluation We evaluated the structures of GST-SigB and MBP-RsbW using IRRAS by comparing the ratio of the amide I and amide II peak intensity (I/II) . In case of GST-SigB, the I/II value became weaker with increasing the heat treatment time. But in case of MBP-RsbW, the I/II value was changed in more comples form during the heat treatment. It suggests that the heat treatment introduced change in structures of both GST-SigB and MBP-RsbW. We found that the thermal stress modulates the interaction between SigB and RsbW and their structures.

2P-056 Identification of interacting domains of the wedge proteins gp11, gp10, gp7, and gp8 of bacteriophage T4 by limited proteolysis

Identification of interacting domains of the wedge proteins gp11, gp10, gp 7, and gp 8 of bacteriophage T4 by limited proteolysis

Moh Lan Yap, Tomoko Nakao, Tatsuya Nagao, Shuji Kanamaru, and Fumio Arisaka (Dept Biomolecular Process Engineering, Graduate School of Bioscience and Biotechnology, Tokyo Institute of Technology

The assembly pathway of the tail of phage $\mathrm{T} 4$ is strictly ordered through sequential interactions of proteins. Seven gene products gp11, gp10, gp7, gp8, gp6, gp53, and gp25 associate sequentially in this order to form a wedge. Gp11 is an exception of this ordered sequential assembly pathway, where it can be added at any stage of the assembly pathway, indicating that its binding site is on the periphery of the baseplate. Gp7 interacts with gp 10 and forms an initial complex of the wedge, followed by the interaction of gp 8, giving rise to a complex of $3: 1: 2$ stoichiometry. In the present study, we isolated gp11, gp10, gp7 and gp 8 and complexes of a number of combinations, namely $(\mathrm{gp} 11)_{3}(\mathrm{gp} 10)_{3},(\mathrm{gp} 10)_{3}(\mathrm{gp} 7)$ and $(\mathrm{gp} \mathrm{10})_{3}(\mathrm{gp} 7)(\mathrm{gp} 8)_{2}$. Sedimentation velocity analyses of the individual proteins and the complexes indicated that they are hydrodynamically homogeneous and that the complexes are stable. Differential limited proteolysis by lysylendopeptidase in the presence and absence of a subunit in the complex were then carried out to elucidate the interacting domains among the subunits: The most striking observation was that the binding of gp7 to gp 10 completely protected gp10 from proteolysis and that the protecting domain of gp 7 that bound to gp 10 was an $\mathrm{N}$-terminal small fragment of $14 \mathrm{kDa}\left(\mathrm{gp} 7^{\mathrm{N} 14 \mathrm{k}}\right.$ ). It also revealed that the binding of gp11 to the domain III of gp10 prevented from digestion of gp10 at Lys 289 . Currently, interacting region of gp 7 with gp 8 is studied by limited proteolysis and crystallization of $(\mathrm{gp} 10)_{3}\left(\mathrm{gp} 7^{\mathrm{N1} 14 \mathrm{k}}\right)$ is underway.

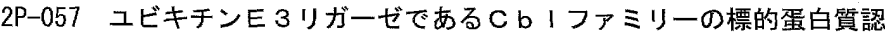
識機構

A target recognition mechanism of human Cbl family ubiquitin E3 ligase Kohei Takeshita(1), Tohnu Tezuka(2,3), Satsuki Nomura(1), Eiki Yamashita(1), Mamoru 